\title{
Biochemical differences among human and animal streptococci of Lancefield group C or group $\mathrm{G}$
}

\author{
ANDROULLA EFSTRATIOU, G. COLMAN, G. HAHN*, J. F. TIMONEY $\dagger$, J. M. BOEUFGRAS $\ddagger$ and \\ D. MONGET $\ddagger$
}

Division of Hospital and Respiratory Infection, Central Public Health Laboratory, London NW9 5HT, *Institut für Hygiene der Bundesanstalt für Milchforschung, Postfach 60 69, D-24121 Kiel, Germany, $\dagger$ Department of Veterinary Science, 108 Gluck Equine Research Center, University of Kentucky, Lexington, Kentucky 40546-0099, USA and $\ddagger$ bioMérieux, 38390, La Balme les Grottes, Vercieu, France

\begin{abstract}
Summary. Pyogenic streptococci of Lancefield group $C$ or group $G$ from human or animal sources were examined with a view to increasing the number of diagnostic tests useful for their differentiation. Human strains of group $\mathrm{G}$ produced L-prolyl-L-arginine aminopeptidase but isolates of Streptococcus equisimilis (group C) did not. Tests for $\alpha$-L-glutamate aminopeptidase together with fermentation of glycogen or sorbitol distinguished $S$. dysgalactiae from strains of $S$. equisimilis isolated from animals. It was confirmed that fermentation tests were helpful in the study of $S$. equi and $S$. zooepidemicus and that enzyme reactions helped distinguish between $S$. canis and the human strains of group G.
\end{abstract}

\section{Introduction}

Streptococci of Lancefield's groups C and G are subdivided conventionally on the basis of their habitat, pathogenicity and biochemical characters. ${ }^{1}$ Four species identifications found useful among strains of group C are: (i) Streptococcus equi, the agent of equine strangles, rarely present in the nose and throat of normal horses and seldom isolated from man; (ii) S. zooepidemicus, the cause of respiratory infections and suppurative disease in many animal speciescarriage is frequent in animals but it is not commonly isolated from man; (iii) $S$. equisimilis, the commonest representative of group $\mathrm{C}$ isolated from $\mathrm{man}^{2}$ in whom it can cause outbreaks of infection $;^{3}$ an organism to which the same name is applied is carried by and causes diseases in domestic animals similar to those associated with $S$. zooepidemicus; ${ }^{1}$ (iv) $S$. dysgalactiae, which can cause bovine mastitis, is a commensal in cattle, and also causes invasive disease in other domestic animals. ${ }^{4}$

Two main taxa have been identified among strains that carry the group $\mathrm{G}$ antigen. One is $S$. canis, which comprises strains isolated from various infections in dogs and cats; strains of group $G$ isolated from mastitis in cattle were included in the original description of this species. ${ }^{5}$ Organisms that are commensals and occasional pathogens in man make up the other. ${ }^{6}$

Some strains of the $S$. milleri group cross-react with either Lancefield group $C$ or $\mathrm{G}$ sera and representatives were included in this study for comparison.
Reactions with trehalose, lactose and sorbitol have been regarded to be of "outstanding value" in the identification of species of group C. ${ }^{1}$ The study of enzymes has also been useful-e.g., $\beta$-glucuronidase, hyaluronidase and fibrinolysin are usually present in human but not bovine strains of group G. ${ }^{6}$ With the exception of $S$. canis, ${ }^{5}$ nucleic acid hybridisation does not provide a method for distinguishing these organisms $;{ }^{7.8}$ this has led to the suggestion that all except $S$. canis can be placed in either $S$. equi or $S$. dysgalactiae aggregates. At times finer subdivisions are necessary and this led us to look for additional diagnostic tests.

\section{Materials and methods}

\section{Bacterial strains}

Forty-three stock strains were screened for significant reactions. Of these, 21 were from the departmental collection of the Central Public Health Laboratory (CPHL) and 22 from the National Collection of Type Cultures, London. They comprised representatives of the taxa listed in the table and five haemolytic strains of $S$. milleri that reacted with the Lancefield $C$ or $G$ grouping reagents. A further 219 strains collected and identified in the UK, Germany or the USA were re-examined at the CPHL. Sixty-three of these were from human clinical material, comprising all 23 strains of S. equisimilis, all 38 human isolates of group $\mathrm{G}$ and two cultures of $S$. zooepidemicus. Except for three cultures of unknown origin-one of $S$. equi and two of $S$. zooepidemicus - the remainder were from animal sources. All 37 strains of $S$. dysgalactiae 
Table. Biochemical properties of 219 streptococcal isolates

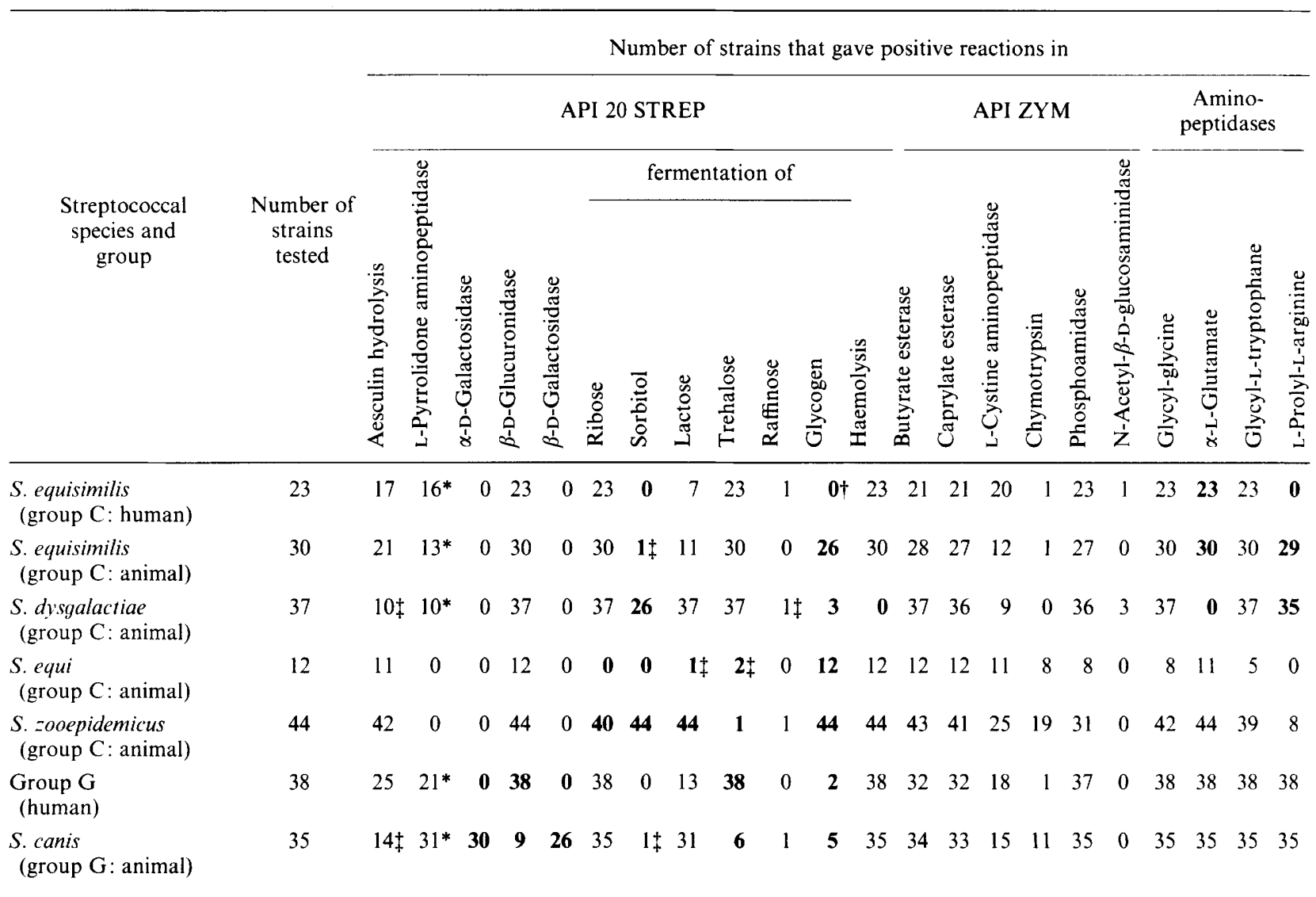

Positive reactions given by all strains. API 20 STREP: alkaline phosphatase, L-leucine aminopeptidase, arginine dihydrolase, starch fermentation. API ZYM : alkaline phosphatase, L-leucine aminopeptidase, L-valine aminopeptidase, acid phosphatase, $\alpha$-D-glucosidase.

Negative reactions with all strains. API 20 STREP: Voges-Proskauer reaction, hippurate hydrolysis, L-arabinose, mannitol and inulin fermentation. API ZYM: lipase-myristate, trypsin, $\alpha$-D-galactosidase, $\beta$-D-glucosidase, $\alpha$-D-mannosidase, $\alpha$-L-fucosidase.

*Trace reactions (see text).

$\dagger$ Useful distinguishing reactions in bold type.

$\ddagger$ Delayed reactions.

and two cultures of $S$. zooepidemicus were of bovine origin. The remaining 116 cultures, with the exception of single isolates from a marten, a sea lion, a zebra and a rhinoceros, were isolated from cats, dogs, pigs or horses of various ages.

\section{Serological grouping}

The Streptex agglutination procedure was used as recommended by the manufacturer (Wellcome Diagnostics, Dartford, Kent) for the detection of group antigens.

\section{Biochemical tests}

Two commercial kits, the API 20 STREP and API ZYM (bioMérieux, Basingstoke, Hants) were employed in the examination of all 262 strains.

The further testing of the 43 stock strains was based on the use of a third package, the API LRA ZYM Research Kit comprising tests for 10 esterases, 20 oxidases and 58 aminopeptidases based, respectively, on the hydrolysis of naphthyl-, para-nitrophenolicand -naphthylamide salts.
The 219 fresh isolates were tested additionally with a specially prepared kit containing 10 substrates to test for four enzymes present in the API LRA ZYM research kit-L-prolyl-L-arginine aminopeptidase, glycyl-L-tryptophane aminopeptidase, $\alpha$-L-glutamate aminopeptidase and glycyl-glycine aminopeptidasetogether with repeat tests for six enzymes incorporated in the API ZYM Kit. The latter were: $\beta$-glucosidase, $\alpha$-glucosidase, $\beta$-glucuronidase, $\beta$-galactosidase, phosphoamidase and $\alpha$-chymotrypsin (or a phenylalanine aminopeptidase).

\section{Results and discussion}

\section{Screening of stock strains}

It was confirmed that haemolytic strains of the $S$. milleri group, which cross-react with Lancefield group $\mathrm{C}$ or group $\mathrm{G}$ sera, could be distinguished readily from the other seven sets of pyogenic streptococci. All five $S$. milleri strains gave the Voges-Proskauer reaction but none formed phosphoamidase. All other cultures gave opposite reactions. Furthermore, the $S$. milleri 
strains neither fermented ribose nor produced $\beta$-glucuronidase, properties that were shared, respectively, with $S$. equi and $S$. canis only.

Six of the reactions covered by the API ZYM kit including phosphoamidase and glucuronidase seemed promising. Production of $\beta$-galactosidase was restricted to $S$. canis; $\beta$-glucosidase is known to be formed by enterococci and some oral streptococci, ${ }^{9}$ so even if not produced by the test strains this could be a test of exclusion. Chymotrypsin was formed by some representatives of $S$. zooepidemicus and $S$. canis. Finally, $\alpha$-glucosidase was formed by the three strains of $S$. milleri placed in group G, but not those of group C.

Although more tests for the presence of particular enzymes were performed with the API LRA ZYM kit most reactions were either uniformly positive or uniformly negative with all stock strains. Four enzymes, namely, glycyl-glycine aminopeptidase, $\alpha$-L-glutamate aminopeptidase, glycyl-L-tryptophane aminopeptidase and L-prolyl-L-arginine seemed worthy of further study.

\section{Testing of fresh isolates}

The results are summarised in the table. Briefly, none formed $\beta$-glucosidase or gave a positive pyrrolidone aminopeptidase reaction. The traces of colour recorded in the table for the latter test were within the range accepted as negative by the manufacturers but are recorded here because they were not distributed evenly among the sets. We believe that the Lancefield group antigen should be determined at an early stage in the identification of these organisms.

The human and animal strains of $S$. equisimilis could be distinguished from each other by glycogen fermentation and production of L-prolyl-L-arginine aminopeptidase. Nearly all the animal strains gave both reactions but none of the human strains gave either. The reaction with glycogen has been reported by others. ${ }^{10}$

$S$. dysgalactiae is known to produce colonies on blood agar surrounded by zones of greening. This was also the case with this collection. Like $S$. equisimilis, strains of $S$. dysgalactiae produced L-prolyl-L-arginine aminopeptidase, but $S$. dysgalactiae strains could be distinguished from $S$. equisimilis because although some fermented sorbitol, none formed $\alpha$-L-glutamate aminopeptidase and few fermented glycogen.

The cultures of $S$. equi differed from all other strains because they failed to exhibit brisk fermentation of any of four substrates-ribose, sorbitol, lactose or trehalose. Delayed reactions were found with one or other of these substrates and have been reported previously. ${ }^{11}$ The well-known pattern of fermentation of sorbitol and lactose but not trehalose by $S$. zooepidemicus was confirmed. Both $S$. equi and $S$. zooepidemicus resembled the animal strains of $S$. equisimilis in fermenting glycogen but differed from that organism and $S$. dysgalactiae in that most strains of both species did not produce L-prolyl-L-arginine aminopeptidase.

Human strains of $S$. equisimilis and the pyogenic group $\mathrm{G}$ streptococci can be distinguished serologically, but not biochemically. ${ }^{7}$ The finding that only the human strains of group $G$ produced L-prolyl-Larginine aminopeptidase provides a useful biochemical test. The human strains of group $\mathrm{G}$ and strains of $S$. canis differed in tests for $\alpha$-D-galactosidase, $\beta$-Dglucuronidase, $\beta$-D-galactosidase and fermentation of trehalose.

The absence of any bovine strains of group $G$ in our collection should be noted. Others have described isolates from dogs and cows as $S$. canis. ${ }^{5}$ However, if the bovine strains are ecologically distinct ${ }^{10}$ it is possible that extended studies would uncover differences. Similarly $S$. equisimilis isolates from animals may comprise several varieties.

This study had the aim of increasing the range of tests that could be used in the identification of streptococci of Lancefield groups C or G, and further work could uncover more of these reactions. For the present, tests for phosphoamidase, $\alpha$-L-glutamate aminopeptidase and L-prolyl-L-arginine aminopeptidase can be added to the repertoire of tests for these organisms that includes Lancefield grouping, fermentation of ribose, sorbitol, lactose, trehalose or glycogen, and the formation of $\alpha$-D-galactosidase, $\beta$-Dglucuronidase, $\beta$-D-galactosidase or L-pyrrolidone aminopeptidase.

\section{References}

1. Stableforth AW. Streptococcal diseases. In: Stableforth AW, Galloway IA (eds) Infectious diseases of animals. Diseases due to bacteria, vol 2. London, Butterworths. 1959: 589-650.

2. Barnham M, Kerby J, Chandler RS, Millar MR. Group C streptococci in human infection: a study of 308 isolates with clinical correlations. Epidemiol Infect 1989; 102: 379-390.

3. Efstratiou A. Outbreaks of human infection caused by pyogenic streptococci of Lancefield groups $\mathrm{C}$ and G. $J \mathrm{Med}$ Microbiol 1989; 29: 207-219.

4. Solberg I. Streptococcus dysgalactiae; a possible disease-pro-

ducing agent in various animal species. Nord Vet Med $1968 ; 20: 26-27$

5. Devriese LA, Hommez J, Kilpper-Bälz R, Schleifer K-H. Streptococcus canis sp. nov.: a species of group G streptococci from animals. Int $J$ Syst Bacteriol 1986; 36: 422-425.

6. Gaunt PN, Seal DV. Group G streptococcal infections. $J$ Infect $1987 ; 15: 5-20$.

7. Farrow JAE, Collins MD. Taxonomic studies on streptococci of serological groups $\mathrm{C}, \mathrm{G}$ and $\mathrm{L}$ and possibly related taxa. Sy'st Appl Microbiol 1984; 5: 483-493.

8. Kilpper-Bälz R, Schleifer K-H. Nucleic acid hybridization and cell wall composition studies of pyogenic streptococci. FEMS Microbiol Lett 1984; 24: 355-364.

9. Kilian M, Mikkelsen L, Henrichsen J. Taxonomic study of 
viridans streptococci: description of Streptococcus gordonii sp. nov. and emended descriptions of Streptococcus sanguis (White and Niven 1946), Streptococcus oralis (Bridge and Sneath 1982), and Streptococcus mitis (Andrewes and Horder 1906). Int J Syst Bacteriol 1989; 39: 471-484.

10. Devriese LA. Streptococcal ecovars associated with different animal species: epidemiological significance of serogroups and biotypes. $J$ Appl Bacteriol 1991; $71: 478-483$.

11. Grant ST, Efstratiou A, Chanter N. Laboratory diagnosis of strangles and the isolation of atypical Streptococcus equi. $V$ et Rec 1993; 133: 215-216.

\title{
ANNOUNCEMENT
}

\author{
Charterhouse Conference \& Communication Co. Ltd \\ Two-day International Conference \\ Challenges in Infection Control
}

Thursday 17th and Friday 18th November 1994 to be held at The Gloucester Hotel, London

Chairmen: Professor S. G. B. Amyes (University of Edinburgh), Professor G. L. French

(Guys Hospital, London), Professor J. E. McGowan (Grady Memorial Hospital, Atlanta),

Dr B. Cookson (Central Public Health).

Topics to include:

- The current state of infections and antibiotics

- The role of antivirals - Aids and the treatment of HIV and other infections

- Growth areas in antibiotic development

- The future of antibiotic therapy

- Cost effectiveness in antibiotic therapy

- Antibacterial chemotherapy - and many more...

For further information about this conference please contact:

The Conference Department

Charterhouse Conference and Communication Co Ltd

35 Cloth Fair, London, EC1A 7JQ

Tel: 0716062435 Fax:071 6062351

\section{BOOKS RECEIVED}

\section{HIV, AIDS and the Brain. Association for Research in} Nervous and Mental Disease Volume 72

Edited by R. W. Price and S. W. Perry. 1993. ISBN 07817-0063-9. Raven Press, New York. Pp. 334. \$125.00.

This book gives a comprehensive and detailed review of recent findings on the neurological complications of human immunodeficiency virus (HIV) infection. It focuses on our current understanding of the direct effect of HIV on the brain, rather than on secondary opportunist infections.

The 17 chapters are divided into three main sections, beginning with background information on the HIV virus and its interaction with the host immune system, particularly the cytokine circuits in the brain and cytotoxic $T$ lymphocytes. William O'Brien's chapter on HIV neurotropism deals with viral biology in manageable bite size pieces suitable even for clinicians with the most rudimentary knowledge of genetics.

The second section forms the bulk of this volume and deals with the latest theory and research in neuropathology and pathogenesis of AIDS dementia complex. Several chapters are devoted to cellular pathology and cytokine expression. There is also a useful chapter on peripheral neuropathy serving as a model for brain pathology. Animal research with simian immunodeficiency virus is reviewed in two chapters. Possibilities for therapeutic intervention are also covered. However, the ultimate question of how HIV causes neurological damage remains unanswered and the final pathways leading to brain injury are undefined. The authors' review illustrates the enormous complexity of the problem. For clinicians, it is an important source of information on current thinking; for academics and scientists, there is plenty of fertile ground for further research.

The final chapters outline the clinical aspects of HIV infection of the brain, including HIV dementia and HIVrelated psychiatric illnesses. One chapter is devoted to neurological diseases in children and infants. Diagnostic evaluations and therapeutic approaches, limited as they currently are, are discussed in detail and are of direct relevance to clinicians.

This is a specialised book most suitable to neuroscientists, virologists and immunologists with special interest in HIV brain diseases. However, it may also be of interest to clinicians coming into frequent contact with neurological complications of HIV disease. It is also a superb reference book for those engaging in HIV research, and should be a very useful addition to any medical library.

M. HUENGSBERG 